2

3

4 Caroline L. Monteil \& Christopher T. Lefevre*

6

7

8

9

10

11

12

13

14

15

16

17

18

19

20

21

22 (1) 2 3 14 5 protists; holobionts; symbiosis; grazing

\title{
Magnetoreception in microorganisms
}

Aix-Marseille University, CNRS, CEA, UMR7265 Institute of Biosciences and Biotechnologies of Aix-Marseille, Saint-Paul-lez-Durance, France

"Correspondence: christopher.lefevre@ @ea.fr (C.T. Lefevre).

Keywords : magnetoreception; biomineralization; magnetosomes; magnetotactic bacteria; 
Magnetoreception is the sense whereby organisms geolocate and navigate in response to the Earth's magnetic field lines. For decades, magnetotactic bacteria have been the only known magnetoreceptive microorganisms. The magnetotactic behaviour of these aquatic prokaryotes is due to the biomineralization of magnetic crystals. While an old report alleged microbial algae with a similar behaviour, recent discoveries have demonstrated the existence of unicellular eukaryotes able to sense the geomagnetic field and revealed different mechanisms and strategies involved in such a sensing. Some ciliates can be magnetically guided after predation of magnetotactic bacteria, while some flagellates acquired this sense through symbiosis with magnetic bacteria.A report has even suggested that some magnetotactic protists could biomineralize magnetic crystals.

\section{Sensing the Earth's magnetic field lines}

Microorganisms can sense environmental features and respond to their fluctuations through different systems of receptors dedicated to, for example, temperature, light, pressure, gravity, energy sources and all sorts of biogenic chemical signals [1-4]. The Earth's magnetic field is no exception and some microorganisms elaborated sensory structures or ecological strategies to exploit its variations. The Earth's core is a permanent magnet whose field vectors point the south magnetic pole away from the north magnetic at every location on the planet but at the equator [5]. The geomagnetic field lines resulting from horizontal and vertical components, every organism able to measure such vector may differentiate the deepness from the surface and the four cardinal directions. During the 11th century, humans learned how to artificially exploit the Earth's magnetic field for navigation with needle compasses [6], while many animals such as birds, fish and insects, naturally have this ability for long-distance migration [7-9]. Magnetoreception emerged in the microbial world too, in which this sense may represent a selective advantage for microorganisms living in spatially fluctuating niches like those of chemically stratified environments. Indeed, the field being locally unchanged, it limits environmental prospection for energy sources and electron acceptors to bidirectional transects instead of a volume [10]. This trait may thus compensate unstable disturbances of vertical chemical gradients and help microorganisms to move toward their optimal local niche. All magnetically sensitive microorganisms described to date associate this sense to a chemoaerotaxis system to swim along chemical and oxygen gradients towards attractants or away repellents. This behaviour, called magnetotaxis, was until recently, observed in magnetotactic bacteria only. However, recent discoveries revealed that some unicellular eukaryotes in different phylogenetic groups are magnetotactic or at least become transiently 
magnetoreceptive by different mechanisms and interactions [11,12]. Here, we propose to review the diversity and different strategies microbes have developed to obtain their magnetic sense.

\section{Bacterial magnetoreception and biomineralization of nanomagnets}

Magnetoreception in bacteria and vertebrates was clearly proven for both at the same period in the late 1970s [13-17]. For vertebrates, elucidating genetic, cellular and biophysical processes involved in magnetoreception remains a challenge in sensory biology for many decades $[18,19]$. Several mechanisms are putatively involved in vertebrates magnetoreception, among which a magnetite-based magnetoreceptor, a light-driven electron transfer reaction in photoreceptors and an electromagnetic induction by electroreceptors [19]. In microorganisms however, only one mechanism is known: the biomineralization of permanent nanomagnets by bacteria within specific organelles called magnetosomes (Figure 1). The mechanistic and genetic basis of magnetic sensing in magnetotactic bacteria (MTB) is the best described so far and was extensively reviewed this last decade [20,21]. Most of our knowledge on magnetosome synthesis comes from genetic and biochemical studies performed on two model strains MSR-1 and AMB-1 of the Magnetospirillum genus. Genomics and genetics identified one cluster of genes of variable size and organization responsible for magnetotaxis, within which an operon, namely the mamAB operon, that is essential for magnetosome formation in all MTB described so far [22,23]. Major progress has been made in the understanding of the genetic basis and biochemical mechanisms involved in magnetosome membrane biogenesis [24], biomineralization [25] and alignment [26]. The alignment in chains of several magnetosomes gives a magnetic moment to the cell along its motility axis, which ensures the parallel orientation with the Earth's magnetic field. Recent biodiversity studies, guided by the development of "omics" technologies, increased our knowledge on the diversity of these bacteria [27-29] and the mechanisms involved in their magnetotactic behaviour [30-32]. While the majority of the lineages described belong to several classes of Proteobacteria, MTB are also distributed in other phyla like the Nitrospirae and the Omnitrophica [33]. Metagenomic data also suggest that some bacteria within the Latescibacteria and Planctomycetes phyla could also form magnetosomes [27,29]. Magnetotactic species produce a diversity of chains with different crystalline structures or chemical composition (magnetite $\mathrm{Fe}_{3} \mathrm{O}_{4}$ or greigite $\mathrm{Fe}_{3} \mathrm{~S}_{4}$ ) that seem to be clade-specific [34]. The processes that led to the polyphyletic distribution of magnetoreception in bacteria in such a wide range of phyla nourished a debate as soon as the first greigite producers were discovered [35]. The timing of magnetosome emergence and the 
history of its diversification over bacteria evolution are a permanent matter of investigation that new genomes and MTB species regularly feed and will fully resolve [27,28,36-38]. The evolutionary steps that led to the emergence of such complex organelle itself and magnetic sensing are so far unknown. MTB evolved different magnetotactic behaviours according to the species [30] with different motility strategies [39] and specific dividing processes [40]. These behaviours represent a selective advantage for these bacteria that find more efficiently their habitat thanks to the inclination of the magnetic field lines [41]. Despite the diversity of the morphologies, motility behaviours, magnetosomes chains and genetic groups, the bacterial magnetic sensing is always associated to bacteria sharing the same niche [29]. This niche is located at the anoxic-microoxic interface of aquatic sediments where the equilibrium of environmental conditions, notably redox, is fragile [30,42]. This zone is the seat of important geochemical processes [42], to which some MTB species were shown to participate in [43].

\section{Magnetically responsive protozoan grazers}

Benthic microbial communities are not only composed of prokaryotes, but also of microbial eukaryotes among which protists and fungi that share the same habitat and need to adapt to the same environmental pressures. After the discovery of the first MTB, arose the hypothesis that magnetotaxis could have evolved in protists thriving in anoxic/microoxic aquatic habitats. In the early years of MTB research, magnetotactic aggregates formed by tens of cells were discovered in a marine lagoon in Brazil [44]. Their multicellularity was rather supporting that these organisms were unlikely bacteria. In fact, those aggregates were the so-called multicellular magnetotactic prokaryotes (MMPs) that represent one of the most interesting and unusual examples of prokaryotic morphology (Figure 1D and E) [45,46]. Later, an AmericanoBrazilian research network opened the way to a new field of research on magnetoreception by observing the first magnetotactic microbial eukaryote [47]. The difficulties to find and collect them have been a huge barrier to the characterization of their magnetoreception for years. Efforts to find them again failed and their study was still limited by the light and electron microscopy analyses carried out in the 80's.

Fifteen years later, populations of diverse magnetic unicellular eukaryotes were observed in the chemocline of the seasonally chemically stratified, coastal Salt Pond, Massachusetts, USA [48]. Biflagellates, dinoflagellates and ciliates were observed among magnetically concentrated microbial communities. A similar observation, from the same environment was reported later [49]. Like magnetotactic bacteria, they migrated and accumulated at the edge of a hanging water droplet in a magnetic field but displayed a different swimming compared to MTB. In these 
125 single-celled eukaryotes, magnetite particles with morphologies and dimensions similar to those of magnetosome-producing bacteria were visualized. However, the origin of these particles was difficult to determine. Based on their observation, endosymbiosis could not be excluded as well as biomineralization by the protist itself, but ingestion of MTB was suggested to be responsible for the magnetic response of some protists, known to be bacterivorous organisms. As many aquatic protists, MTB grazers (i.e. protists that predate MTB) are facultative anaerobes capable of both aerobic and anaerobic growth, which should allow them to easily predate MTB. In Salt Pond, it was shown that the magnetically responsive protists were more abundant during the early and late season of stratification, when the chemocline narrows and MTB concentrate in a smaller volume [49], indicating a correlation between concentration of MTB and that of magnetically responsive protists. Moreover, when the magnetotactic protists were magnetically concentrated at the edge of a droplet where MTB aggregate, they appeared to exhibit a slow looping swimming motion that could be associated to a predatory behaviour [49].

By feeding a predatory ciliate with magnetically purified $\mathrm{Ca}$. Magnetoglobus multicellularis, it has been shown that greigite magnetosomes could be dissolved within the acidic vacuoles of the ciliate [50]. In this study, it was not possible to state if this behaviour occurs in environmental conditions and if magnetoreception could arise from the interaction. Recently, this behaviour was investigated in natural populations of protists and MTB, and it was shown that ciliates affiliated to the genus Uronema (Stramenopiles-Alveolates-Rhizaria, SAR group) were able to ingest hundreds of MTB into acidic vacuoles to progressively become sensitive to the variations of the magnetic fields in the same way than MTB (Figure 2A and B) [11]. The magnetic response in MTB-grazers is certainly not encoded in the eukaryote genome, but this behaviour seems to facilitate movement of grazers towards the prey biomass. It is still unclear if a chemotaxis is also involved and if this interaction has been selected over the course of the protist evolution. However, the directed grazing towards these preys specifically and the magnetic response suggest that this interaction is widespread among marine and freshwater heterotrophic flagellates and ciliates (Figure 2). MTB-grazers seem to have evolved different strategies for the internalization of MTB and their magnetosome chains: (i) sequestration in food vacuoles (Figure 2A and B), (ii) accumulation in a specific location in the cell (Figure 2C and D), and (iii) an apparently random storage in the cell (Figure 2E) [11,48,50]. These magnetically responsive protists also appear to have evolved different strategies to deal with iron toxicity with (i) the egestion of magnetic inclusions where magnetosomes accumulated [48] and (ii) the progressive dissolution of magnetosomes until the colloidal iron would be 
expelled from the protistan cell via the cytoproct [11,50,51]. Protists that feed on MTB have been proposed to play a significant role in iron cycling $[11,48,49]$. It was shown that digestion of colloidal iron in the food vacuoles of protists during grazing of particulate and colloidal matter could generate more bioavailable iron for other species, such as phytoplankton $[52,53]$. Thus, MTB grazing could be involve in recycling particulate iron back to a bioavailable form in the environment through dissolution [54].

\section{Symbiotic origin of magnetoreception in protists}

Long neglected, mutualistic symbiosis and cooperation are today recognized as major diversification forces in the same way as competition for resources and natural selection have been for centuries [55]. These concepts even became central in our understanding of the mechanisms and strategies governing species interactions and adaptation in innumerable biological systems. Mutualistic symbioses between prokaryotes - macroscopic eukaryotes are the best examples. They have been particularly well documented for insects, plants or corals, for which studies have shown the importance of certain highly specialized algae, bacteria and fungi on host nutrition and reproduction [56-58]. These symbioses are also extremely diverse and abundant between unicellular eukaryotes and prokaryotes, even though less characterized [59]. Some of them are even the foundation of the evolutionary scenarios on organelle acquisition and eukaryogenesis [60]. Symbiotic associations between protists and bacteria or archaea are common in anoxic marine sediments [61]. Prokaryotic symbionts can be associated with their protist hosts as ectosymbionts (attached to the host surface) or as endosymbionts (located beneath the host cell membrane). These symbionts can expand the host niche by complementing host metabolism, improving its motility or adaptation to new conditions.

The recent discovery of magnetotactic holobionts changed our vision of magnetoreception in unicellular eukaryotes. A magnetotactic protist belonging to the Euglenozoa, Excavates, was observed in marine sediments worldwide [12,62]. Its magnetoreception was the result of a cooperation with ectosymbiotic bacteria with whom they live in a mutualistic symbiosis (Figure 3) [12]. Again, magnetoreception is magnetite-based and originates from prokaryotes biomineralizing magnetosomes chains. Unlike MTB-grazers, the sensing and geolocalization in this biological system benefits to both organisms thanks to the long-term cooperation established with the host. Microscopy and genomic analyses indicate these ectosymbiotic bacteria are not magnetotactic like MTB in the sense they do not move by means of their own flagella and do not sense chemical gradients; they can be considered only as magnetic. With the protistan host, magnetic ectosymbiotic bacteria (MEB) form a microbial holobiont acting 
as a supramicroorganism and an ecological unit. Hundreds of these MEB, all belonging to a single strain of the Deltaproteobacteria class, are aligned parallel to the host motility axis (Figure 3A and B) and magnetically orient it. Surprisingly, it was experimentally proven that this magnetic guidance was influenced by oxygen concentrations while genome sequencing showed that MEB could not sense chemical gradients. It is thus likely that holobiont magnetotaxis was collectively ensured thanks to the biflagellate chemotaxis. The partners' interdependency relies also on metabolic exchanges, among which some of them could be identified based on the host ultrastructure and MEB genome. Their syntrophy is based on the transfer of molecular hydrogen from the host to the MEB that use it to reduce sulphate. Protists are bacteria predators and ferment organic matter into energy thanks to mitochondrion-like organelles called hydrogenosomes [63]. This process generates ATP and by products like $\mathrm{CO}_{2}$, acetate and $\mathrm{H}_{2}$ that is diffused through the membrane plasma. Other chemicals might be exchanged but need to be identified through isotope probing and comparative genomics. For example, genomic data indicate that acetate and $\mathrm{CO}_{2}$ are likely used by the MEB as well [12].

\section{Magnetic biomineralization in protists?}

The search for biomineralizing magnetic microbial eukaryotes has become an endeavour for many researchers as soon as Torres de Ajaujo and coworkers reported in 1986 the magnetotactic algae with many magnetite particles organized in chains [47]. It was clear that the magnetoreception of these microorganisms was magnetite-based similarly to MTB. Although the chains appeared to be located in or near the cell wall, authors could not identify their organization relative to each other and from the flagellate motility axis. With the limitation of the imaging techniques and DNA typing at that time, many questions remained unanswered, making it hard to conclude about the origin of their magnetoreception. No ultrastructural feature could inform on the presence or the absence of ecto- or endo-symbiotic bacteria even though bullet-shaped magnetosomes looked like those of Deltaproteobacteria [34]. These flagellated protists, tentatively identified as Anisonema platysomum (Euglenophyceae, Euglenozoa, Excavata) based on morphological criteria, were not observed again in decades after their first isolation in a coastal mangrove swamp in northeastern Brazil.

In 2019, a new report revivals the hypothesis of a biomineralization-based magnetoreception in unicellular eukaryotes [64]. Uncultured single-celled eukaryotic flagellates from two different Brazilian freshwater sites were observed harbouring anisotropic bullet-shaped magnetite magnetosomes aligned in complex aggregations of multiple chains within the cell. Light and transmission electron microscopy (TEM) images of these magnetotactic microbes were not 
decisive enough to allow their identification and no genotyping or genome sequencing was performed. Their size, morphology and the multiple flagella at the anterior pole of the cells attested that the protist belongs to another group of that previously observed 30 years before. Because of the magnetosomes size two times longer than bacterial magnetosomes and the absence of specific micro-compartments typical of a bacterial cell or digestive vacuoles, observations rather supported that the biomineralization is performed by the eukaryote itself. However, contrasted TEM cross sections are missing to validate the ultrastructure of the magnetotactic protist and the relative position of the magnetosomes chains. If such result was validated, magnetoreception could have arose from an ancient endosymbiosis event with an MTB, but also from a secondary lateral gene transfer of the whole magnetosome genes cluster. Even if these scenarios are those the most plausible and parsimonious, it is not to exclude that magnetic biomineralization emerged independently a second time in eukaryotes and converged toward the same function. Future research, such as the identification of the genetic determinants involved in magnetosomes formation, will help to validate that magnetosome magnetite biomineralization occurs in this protist.

\section{Concluding Remarks and Future Prospects}

The latest discoveries have unravelled a broader diversity of magnetoreceptive microorganisms in the Eukarya domain than previously thought. Advances in single-cell sorting and genomics are opening up a path to study their ultrastructural, phylogenetic and biological characterization. So far, microbial magnetoreception seems to be restricted to aquatic microorganisms and to be systematically coupled to magnetotaxis, which includes biomineralization of magnetic particles, chemo-aerotaxis and a motility response. But two other ecological strategies were shown to be associated indirectly with magnetoreception in microorganisms: predation of magnetotactic bacteria and symbiosis with magnetic bacteria (Figure 4). The distribution of this function among microorganisms inhabiting anoxic/microoxic habitats strengthens the idea that it represents a key adaptation to these habitats. Although our knowledge on the ecology, diversity and evolution of magnetoreceptive microorganisms have dramatically increased these recent years [29,37,65], many important questions remain unanswered and should be addressed in future work (see Outstanding Questions). Only one prokaryotic origin has been formally demonstrated, and a possible independent emergence in eukaryotes needs to be investigated. The discovery of magnetotactic protists specifically, paves the way to a new field of research at the interface of many disciplines. It is unclear yet in which extent this trait is widespread among benthic protists since the classical isolation protocols and methods still need to be 
adapted to these organisms. Furthermore, all magnetoreceptive microorganisms identified to date are those coupling magnetoreception and geolocalization to navigation. But other biological systems showed that geolocalization may be used for a purpose other than navigation, as some immobile fungi that use gravity instead to sense verticality and direct the spread of spores [66]. Thus, beyond exploring the diversity of magnetotactic organisms, alternative protocols should be developed to isolate magnetoreceptive microorganisms that are not magnetotactic. Exploration of new habitats along with the application of new material and methods for the observation of magnetically responsive organisms will certainly answer this question and many others that the scientific community is currently enthusiastic about.

The discovery of microorganisms able of magnetoreception is also particularly appealing for some scientists that search for the origin of magnetoreception in macroorganisms like migratory birds or fish. Indeed, for non-specialists in evolutionary biology, it is easy to mistakenly think that modern lineages of microbial eukaryotes reflect ancestral forms of these. As a consequence, it was speculated that ancient prokaryotic or eukaryotic forms of magnetosomes or even endosymbiotic MTB could be at the origin of magnetoreception in animals $[67,68]$. The possibility of a symbiosis between a magnetotactic bacterium that would be at the origin of magnetoreception remains very speculative, as there is no evidence of the presence of MTB in symbiosis with such animal. For example, magnetite biomineralization mechanisms have been identified for some macroorganisms such as chitons or honeybees and would involve ferritin deposition [71,72], not magnetosome formation. Then, despites the report of the presence of magnetotactic symbionts [69], closely related to the free-living MTB strain SS-5 [70], within the marine bivalve Thyasira $c f$. gouldi, this association is likely more related to predation. Indeed, the magnetotactic cells in their host lose the integrity of their magnetosome chain and possibly their flagellum. Moreover, the bivalves appeared to not use magnetotactic bacteria for magnetoreception while bacteria do not seem to take any advantage to be in the host. Thus, there is no evidence of a connexion between magnetotactic bacteria and magnetoreception in animals so far. We believe that advanced methods in imaging and genomics will contribute in the future to decipher some of the enigma about the diversity of the magnetoreceptive mechanisms, its emergence and evolution in micro- and macroorganisms.

\section{Acknowledgments}

We thank the French National Research Agency (ANR-16-TERC-0025-01 and ANR-18-CE310003), the New Zealand Ministry for Business, Innovation and Employment (grant no. 
LVLX1703) and all our collaborators and other researchers that contribute to make this field of research so exciting and in perpetual progression.

296

\section{References}

298

299

300

301

302

303

304

305

306

307

308

309

310

311

312

313

1 Bryant, D.A. and Frigaard, N.-U. (2006) Prokaryotic photosynthesis and phototrophy illuminated. Trends Microbiol. 14, 488-496

2 Walsby, A.E. (1994) Gas vesicles. Microbiol. Rev. 58, 94-144

3 Wadhams, G.H. and Armitage, J.P. (2004) Making sense of it all: bacterial chemotaxis. Nat. Rev. Mol. Cell Biol. 5, 1024-1037

4 Shivaji, S. and Prakash, J.S.S. (2010) How do bacteria sense and respond to low temperature? Arch. Microbiol. 192, 85-95

5 Guyodo, Y. and Valet, J.P. (1999) Global changes in intensity of the Earth's magnetic field during the past $800 \mathrm{kyr}$. Nature 399, 249-252

6 Merrill, R.T. and McElhinny, M.W. (1983) The Earth's Magnetic Field: Its History, Origin, and Planetary Perspective, Academic Press.

7 Wiltschko, R. and Wiltschko, W. (2012) Magnetoreception. Adv. Exp. Med. Biol. 739, 126141

8 Clites, B.L. and Pierce, J.T. (2017) Identifying Cellular and Molecular Mechanisms for Magnetosensation. Annu. Rev. Neurosci. 40, 231-250

9 Begall, S. et al. (2014) Magnetoreception in Mammals. In Advances in the Study of Behavior, Vol 4646 (Naguib, M. et al., eds), pp. 45-88, Elsevier Academic Press Inc

10 Klumpp, S. et al. (2019) Swimming with magnets: From biological organisms to synthetic devices. Phys. Rep. 789, 1-54

11 Monteil, C.L. et al. (2018) Accumulation and Dissolution of Magnetite Crystals in a Magnetically Responsive Ciliate. Appl. Environ. Microbiol. 84, pii: e02865-17

12 Monteil, C.L. et al. (2019) Ectosymbiotic bacteria at the origin of magnetoreception in a marine protist. Nat. Microbiol. DOI: 10.1038/s41564-019-0432-7

13 Blakemore, R. (1975) Magnetotactic bacteria. Science 190, 377-379

14 Walcott, C. et al. (1979) Pigeons have magnets. Science 205, 1027-1029

15 Gould, J.L. et al. (1978) Bees have magnetic remanence. Science 201, 1026-1028

16 Frankel, R. et al. (1979) Magnetite in Freshwater Magnetotactic Bacteria. Science 203, 1355-1356

17 Bellini, S. (2009) On a unique behavior of freshwater bacteria. Chin. J. Oceanol. Limnol. $27,3-5$ 
18 Mouritsen, H. (2018) Long-distance navigation and magnetoreception in migratory animals. Nature 558, 50-59

19 Nordmann, G.C. et al. (2017) Magnetoreception-A sense without a receptor. PLOS Biol. $15, \mathrm{e} 2003234$

20 Uebe, R. and Schüler, D. (2016) Magnetosome biogenesis in magnetotactic bacteria. Nat. Rev. Microbiol. 14, 621-637

21 Grant, C.R. et al. (2018) Organelle Formation in Bacteria and Archaea. Annu. Rev. Cell Dev. Biol. 34, 217-238

22 Lohsse, A. et al. (2011) Functional analysis of the magnetosome island in Magnetospirillum gryphiswaldense: the mamAB operon is sufficient for magnetite biomineralization. PloS One 6, e25561

23 Lefèvre, C.T. et al. (2013) Comparative genomic analysis of magnetotactic bacteria from the Deltaproteobacteria provides new insights into magnetite and greigite magnetosome genes required for magnetotaxis. Environ. Microbiol. 15, 2712-2735

24 Raschdorf, O. et al. (2016) Genetic and Ultrastructural Analysis Reveals the Key Players and Initial Steps of Bacterial Magnetosome Membrane Biogenesis. PLOS Genet. 12, e1006101

25 Hershey, D.M. et al. (2016) MamO Is a repurposed serine protease that promotes magnetite biomineralization through direct transition metal binding in magnetotactic bacteria. PLoS Biol. 14, e1002402

26 Toro-Nahuelpan, M. et al. (2019) MamY is a membrane-bound protein that aligns magnetosomes and the motility axis of helical magnetotactic bacteria. Nat. Microbiol. DOI: 10.1038/s41564-019-0512-8

27 Lin, W. et al. (2018) Genomic expansion of magnetotactic bacteria reveals an early common origin of magnetotaxis with lineage-specific evolution. ISME J. 12, 1508-1519

28 Lin, W. et al. (2017) Origin of microbial biomineralization and magnetotaxis during the Archean. Proc. Natl. Acad. Sci. U. S. A. 114, 2171-2176

29 Lin, W. et al. (2017) Diversity and ecology of and biomineralization by magnetotactic bacteria. Environ. Microbiol. Rep. 9, 345-356

30 Lefèvre, C.T. et al. (2014) Diversity of magneto-aerotactic behaviors and oxygen sensing mechanisms in cultured magnetotactic bacteria. Biophys. J. 107, 527-538

31 González, L.M. et al. (2015) Sudden motility reversal indicates sensing of magnetic field gradients in Magnetospirillum magneticum AMB-1 strain. ISME J. 9, 1399-1409 
32 Popp, F. et al. (2014) Polarity of bacterial magnetotaxis is controlled by aerotaxis through a common sensory pathway. Nat. Commun. 5, 5398

33 Kolinko, S. et al. (2016) Single-cell genomics of uncultivated deep-branching magnetotactic bacteria reveals a conserved set of magnetosome genes. Environ. Microbiol. $18,21-37$

34 Pósfai, M. et al. (2013) Phylogenetic significance of composition and crystal morphology of magnetosome minerals. Front. Microbiol. 4, 344

35 DeLong, E.F. et al. (1993) Multiple evolutionary origins of magnetotaxis in bacteria. Science 259, 803-806

36 Lefèvre, C.T. et al. (2013) Monophyletic origin of magnetotaxis and the first magnetosomes. Environ. Microbiol. 15, 2267-2274

37 Lefèvre, C.T. and Wu, L.F. (2013) Evolution of the bacterial organelle responsible for magnetotaxis. Trends Microbiol. 21, 534-543

38 Monteil, C.L. et al. (2018) Genomic study of a novel magnetotactic Alphaproteobacteria uncovers the multiple ancestry of magnetotaxis. Environ. Microbiol. 20, 4415-4430

39 Murat, D. et al. (2015) Opposite and coordinated rotation of amphitrichous flagella governs oriented swimming and reversals in a magnetotactic spirillum. J. Bacteriol. 197, 3275-3282

40 Lefèvre, C.T. et al. (2015) Positioning the Flagellum at the Center of a Dividing Cell To Combine Bacterial Division with Magnetic Polarity. mBio 6, e02286

41 Bennet, M. et al. (2014) Influence of Magnetic Fields on Magneto-Aerotaxis. PLoS ONE 9, e101150

42 Brune, A. et al. (2000) Life at the oxic-anoxic interface: microbial activities and adaptations. FEMS Microbiol. Rev. 24, 691-710

43 Rivas-Lamelo, S. et al. (2017) Magnetotactic bacteria as a new model for P sequestration in the ferruginous Lake Pavin. Geochem. Perspect. Lett. 5, 35-41

44 Esquivel, D. et al. (1983) Magnetotactic Microorganisms in the Rio-De-Janeiro Region. Biol. Cell 47, 227-233

45 Abreu, F. et al. (2013) Cell adhesion, multicellular morphology, and magnetosome distribution in the multicellular magnetotactic prokaryote Candidatus Magnetoglobus multicellularis. Microsc. Microanal. Off. J. Microsc. Soc. Am. Microbeam Anal. Soc. Microsc. Soc. Can. 3, 1-9

46 Qian, X.-X. et al. (2019) Juxtaposed membranes underpin cellular adhesion and display unilateral cell division of multicellular magnetotactic prokaryotes. Environ. Microbiol. DOI: $10.1111 / 1462-2920.14710$ 
47 Torres de Araujo, F.F. et al. (1986) Magnetite and magnetotaxis in algae. Biophys. J. 50, 375-378

48 Bazylinski, D.A. et al. (2000) Occurrence and distribution of diverse populations of magnetic protists in a chemically stratified coastal salt pond. Chem. Geol. 169, 319-328

49 Simmons, S.L. and Edwards, K.J. (2006) Geobiology of magnetotactic bacteria. In Magnetoreception and Magnetosomes in Bacteria pp. 77-102, Springer, Berlin, Heidelberg

50 Martins, J.L. et al. (2007) Grazing protozoa and magnetosome dissolution in magnetotactic bacteria. Environ. Microbiol. 9, 2775-2781

51 Allen, R. (1974) Food vacuole membrane growth with microtubule-associated membranetransport in Paramecium. J. Cell Biol. 63, 904-922

52 Barbeau, K. et al. (1996) Role of protozoan grazing in relieving iron limitation of phytoplankton. Nature 380, 380061a0

53 Pernthaler, J. (2005) Predation on prokaryotes in the water column and its ecological implications. Nat. Rev. Microbiol. 3, 537-546

54 Sherr, E.B. and Sherr, B.F. (2002) Significance of predation by protists in aquatic microbial food webs. Antonie Van Leeuwenhoek 81, 293-308

55 Margulis, L. and Fester, R. (1991) Symbiosis as a Source of Evolutionary Innovation: Speciation and Morphogenesis, MIT Press.

56 Rowan, R. and Knowlton, N. (1995) Intraspecific diversity and ecological zonation in coral algal symbiosis. Proc. Natl. Acad. Sci. U. S. A. 92, 2850-2853

57 Engel, P. and Moran, N.A. (2013) The gut microbiota of insects - diversity in structure and function. Fems Microbiol. Rev. 37, 699-735

58 Vandenkoornhuyse, P. et al. (2015) The importance of the microbiome of the plant holobiont. New Phytol. 206, 1196-1206

59 López-García, P. et al. (2017) Symbiosis in eukaryotic evolution. J. Theor. Biol. 434, 2033

60 López-García, P. and Moreira, D. (2015) Open questions on the origin of eukaryotes. Trends Ecol. Evol. 30, 697-708

61 Bernhard, J.M. et al. (2000) The Santa Barbara Basin is a symbiosis oasis. Nature 403, 7780

62 Yubuki, N. and Leander, B.S. (2018) Diversity and Evolutionary History of the Symbiontida (Euglenozoa). Front. Ecol. Evol. 6, 100. doi: 10.3389/fevo.2018.00100

63 Müller, M. et al. (2012) Biochemistry and evolution of anaerobic energy metabolism in eukaryotes. Microbiol. Mol. Biol. Rev. MMBR 76, 444-495 
64 Leão, P. et al. Magnetosome magnetite biomineralization in a flagellated protist: evidence for an early evolutionary origin for magnetoreception in eukaryotes. Environ. Microbiol. doi: 10.1111/1462-2920.14711

65 Lefèvre, C.T. and Bazylinski, D.A. (2013) Ecology, diversity, and evolution of magnetotactic bacteria. Microbiol Mol Biol Rev 77, 497-526

66 Nguyen, T.A. et al. (2018) Evolutionary novelty in gravity sensing through horizontal gene transfer and high-order protein assembly. PLOS Biol. 16, e2004920

67 Kirschvink, J.L. et al. (2001) Magnetite-based magnetoreception. Curr. Opin. Neurobiol. $11,462-467$

68 Natan, E. and Vortman, Y. (2017) The symbiotic magnetic-sensing hypothesis: do Magnetotactic Bacteria underlie the magnetic sensing capability of animals? Mov. Ecol. 5, 22. doi: 10.1186/s40462-017-0113-1

69 Dufour, S.C. et al. (2014) Magnetosome-containing bacteria living as symbionts of bivalves. ISME J. 8, 2453-2462

70 Lefèvre, C.T. et al. (2012) Novel magnetite-producing magnetotactic bacteria belonging to the Gammaproteobacteria. ISME J. 6, 440-450

71 Shaw, J.A. et al. (2009) Ultrastructure of the epithelial cells associated with tooth biomineralization in the chiton Acanthopleura hirtosa. Microsc Microanal, 15, 154-65

$72 \mathrm{Hsu}$, C.Y. and Chan, Y.P. (2011) Identification and localization of proteins associated with biomineralization in the iron deposition vesicles of honeybees (Apis mellifera). PLoS One. 6, e19088 
453 Biomineralization: biological process by which eukaryotes and prokaryotes are able to 454 produce minerals.

455 Chemocline: horizontal layer formed in aquatic habitats by a strong vertical chemistry gradient 456 that marks the boundary between two contrasted chemical environments.

457 Cytoproct: special pore in the pellicle of ciliates used for exocytosis and membrane recycling.

458 Eukaryogenesis: evolutionary transition at the origin of the eukaryotic cell.

459 Grazing: feeding strategy that some protozoa evolved to feed on microorganisms.

460 Hydrogenosomes: double membrane-bounded organelles of mitochondrial ancestry found in 461 some anaerobic eukaryotes. They are involved in catabolic processes that produce molecular hydrogen, acetate, carbon dioxide and ATP.

Holobiont: Assemblage of different species living in symbiosis that forms an ecological unit. Magnetosomes: prokaryotic organelles composed of nano-sized, magnetic, iron-mineral crystals, enveloped by a biological membrane. Usually arranged in chains within the cell, they provide the cell with a permanent magnetic dipole and allow magnetic sensing.

467 Magnetotaxis: behaviour of some motile aquatic bacteria that align passively along Earth's magnetic field lines while they swim to facilitate their navigation towards their preferred habitat.

Mutualistic symbiosis: long-term relationship between different species living in symbiosis in which all partners benefit from the biological interaction.

472 Protists: unicellular eukaryotic microorganisms (cells containing a nucleus). They do not refer 473 to a taxonomic unit and are polyphyletically distributed into the Eukarya domain.

474 Protozoa: heterotrophic unicellular eukaryotes that feed on organic matter such as other microorganisms.

Polyphyletic distribution: Distribution of a trait or organisms in different taxonomic groups that are not related by a direct common ancestor.

Symbiosis: defines a long-term physical coexistence of two or more species, in which at least one partner is dependent on the others, no matter what the effect of the interaction on the partner's fitness.

Syntrophy: trophic interdependency of two symbiotic species. Also referred as a mutualistic metabolism in which partner's metabolisms rely on the exchange of metabolic products of each other's. 
(A)

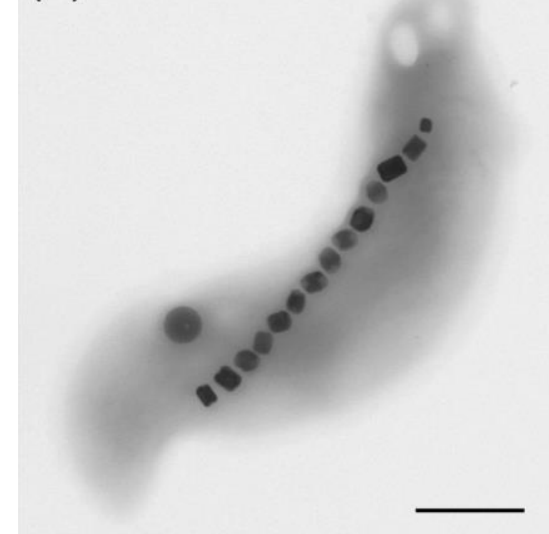

(D)

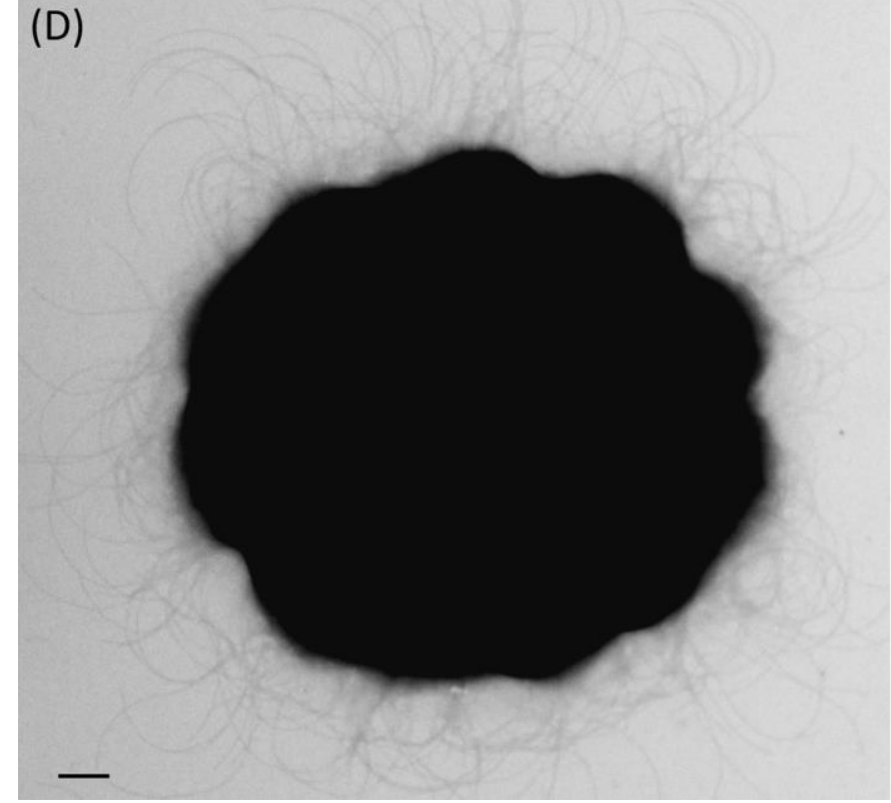

(B)
(C)

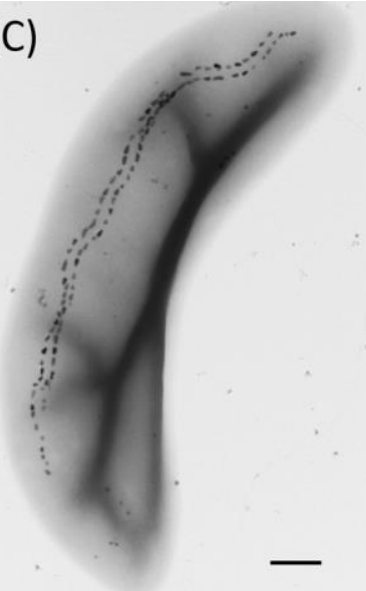

(E)

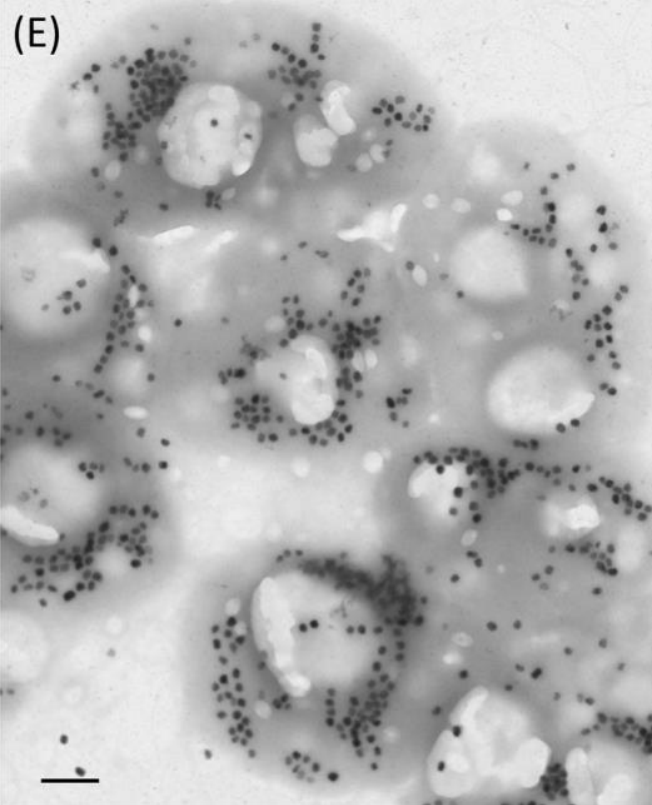

487

488

489

490

491

492

493

494

495

Figure 1. Transmission electron microscope images of magnetotactic bacteria.

Magnetotactic spirillum (A), cocci (B) and curved rod (C) isolated from the freshwater Lake Pavin, France. Intact (D) and disaggregated (D) multicellular magnetotactic prokaryote (MMP) isolated from the Mediterranean Sea in Carry-le-Rouet, France. Magnetotactic cells in (A-C) produce elongated prismatic (A and B) or bullet-shaped (C) magnetite magnetosomes while the MMP (E) produce octahedral greigite magnetosomes. Scale bars represent $0.5 \mu \mathrm{m}$. 

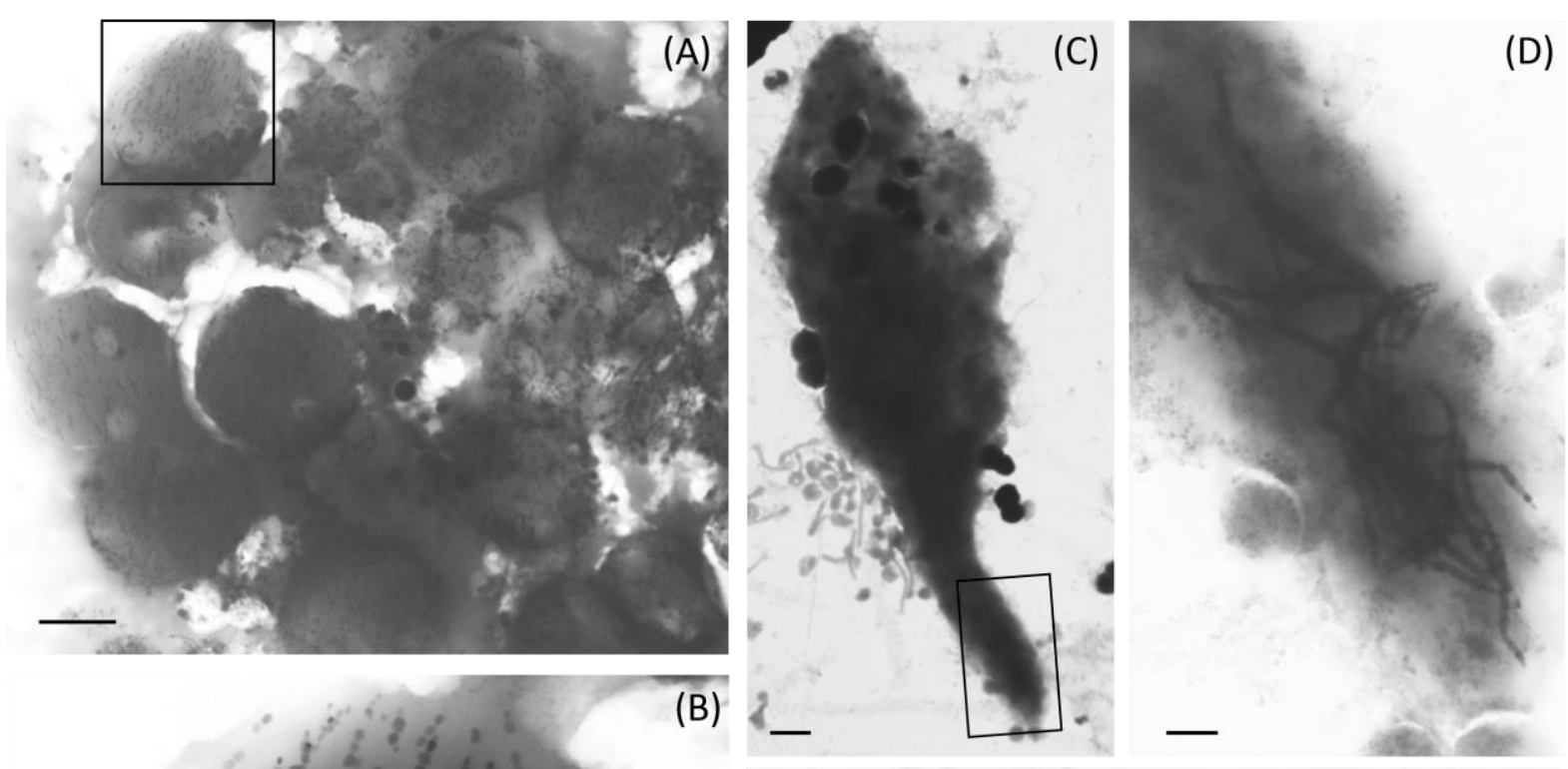

(D)

(E)
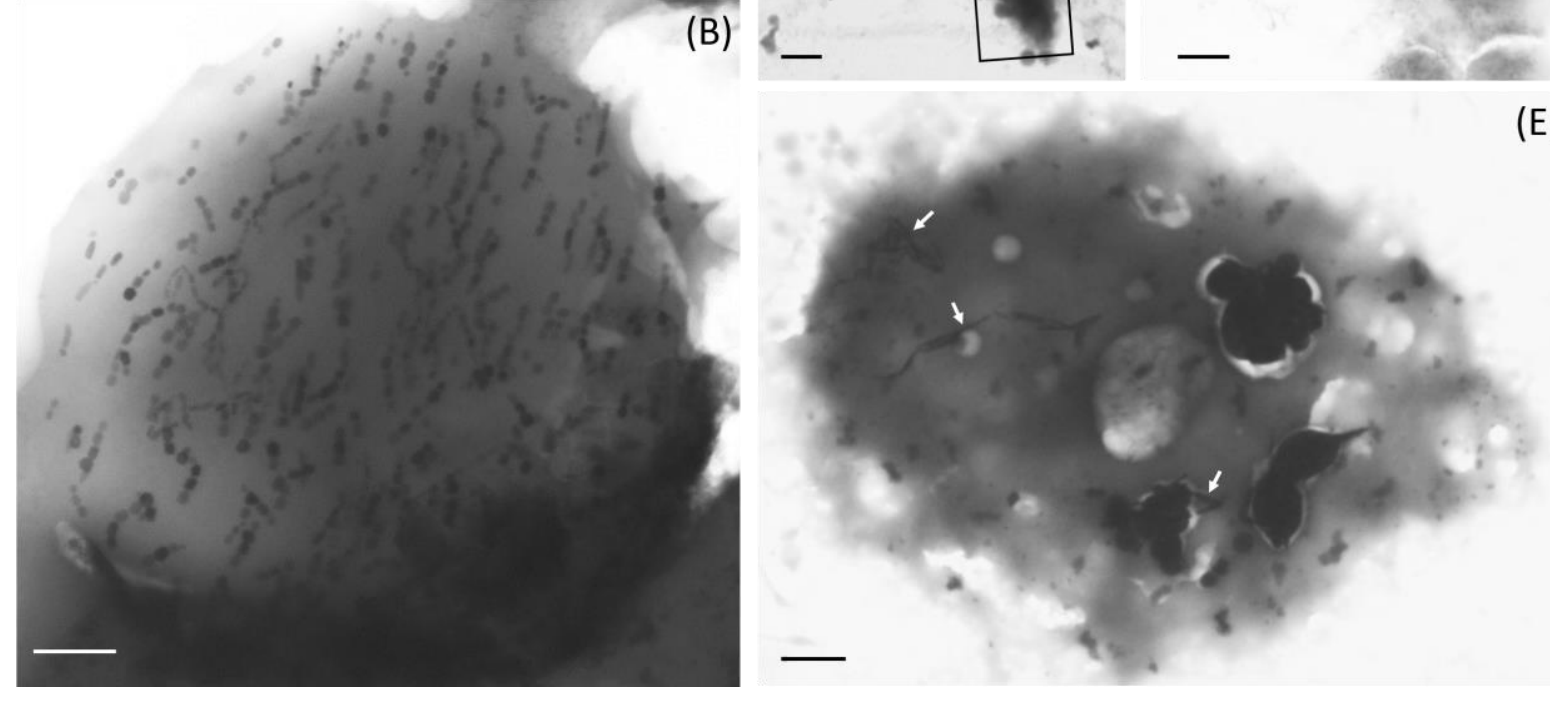

497 Figure 2. Transmission electron microscope images of magnetic ciliates that grazed magnetosomes in digestive vacuoles. The black frame in (A) shows where the higher magnification image in (B) was taken. (C-D) Ciliate that accumulates magnetosomes in a specific location within its cytoplasm. The black frame in (C) shows where the higher magnification image in (D) was taken. (E) Ciliate that randomly stores grazed magnetotactic bacteria inside its cell. Scale bars represent $2 \mu \mathrm{m}$ (A, C and E) and $0.5 \mu \mathrm{m}$ (B and D). 

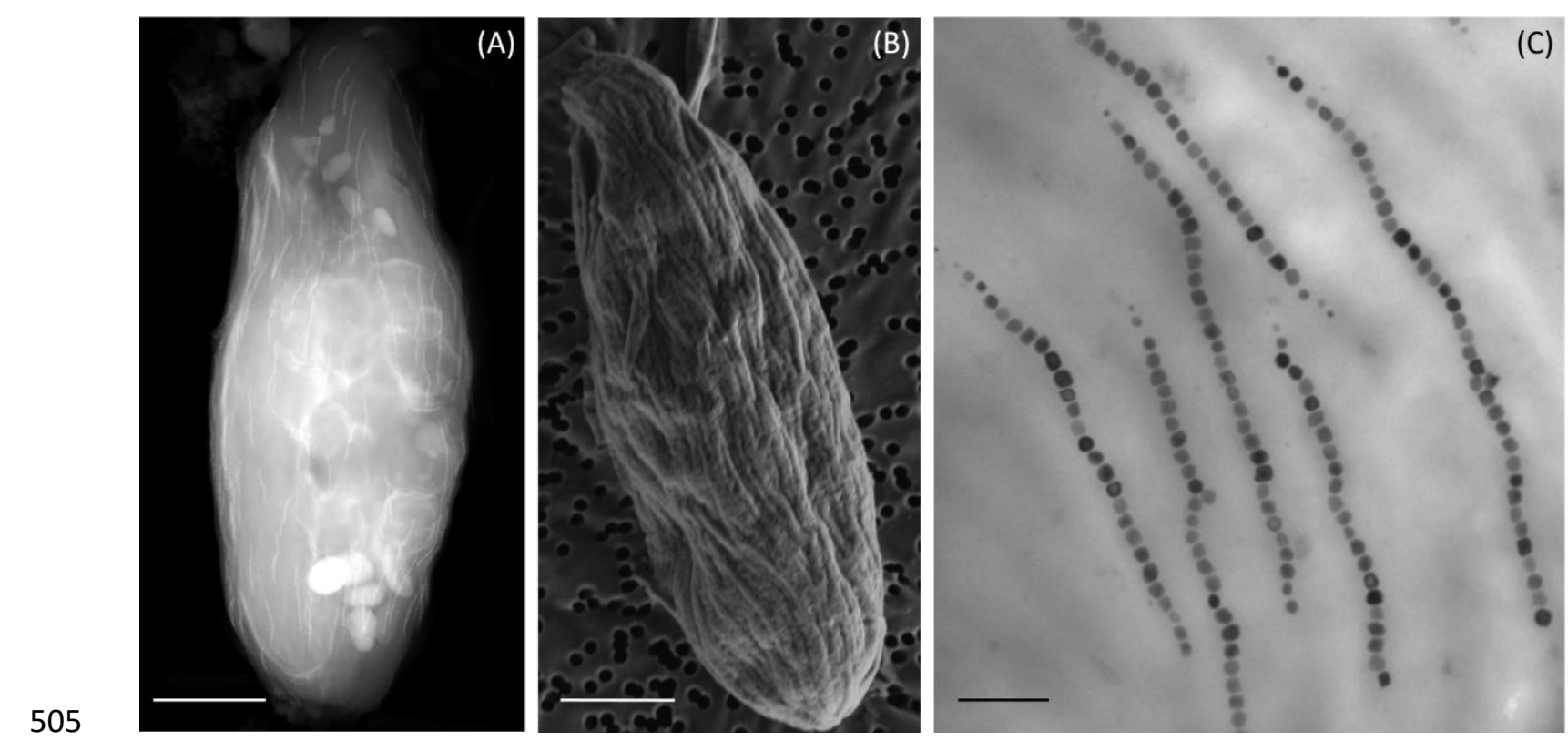

506 Figure 3. Electron microscope images of magnetotactic holobionts isolated from the

507 Mediterranean Sea. High angle annular dark field scanning transmission electron microscope

508 (A) and scanning transmission electron microscope (B) images of single magnetotactic 509 holobionts showing the presence of hundreds of bacteria surrounded a protistan cell. (C)

510 Transmission electron microscope image of several magnetosome chains produced by magnetic 511 ectosymbiotic bacteria allowing magnetoreception of the protist. Scale bars represent $2 \mu \mathrm{m}$ (A 512 and B) and $0.5 \mu \mathrm{m}(\mathrm{C})$. (A and B) Image courtesy of N. Menguy and K. Benzerara, respectively. 


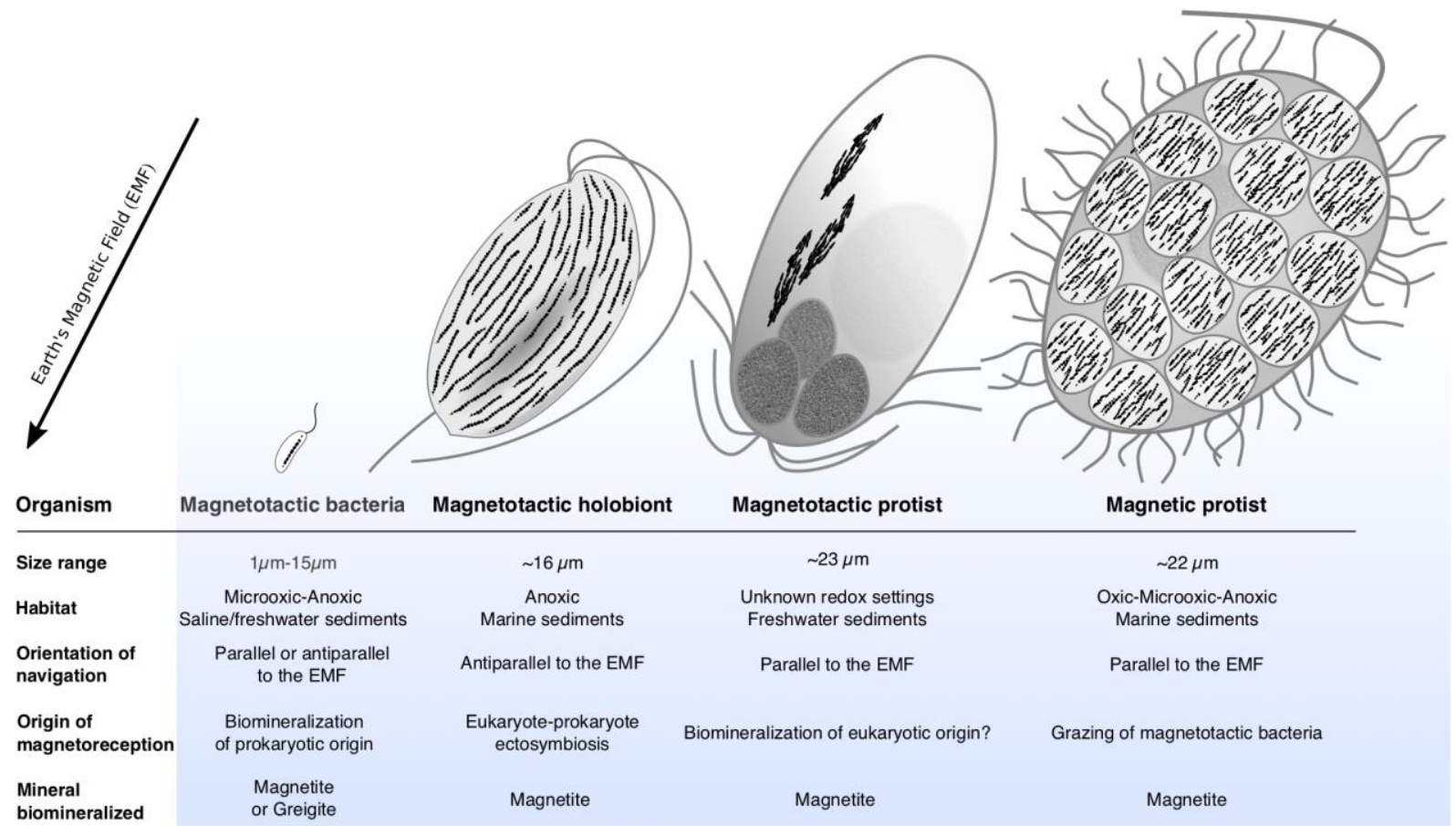

515 Figure 4. Schematic comparison of magnetoreceptive microorganisms morphologies and

516 magnetic behaviours. Magnetosomes chains are represented in black. Organism's anterior-

517 posterior orientation is given relative to the Earth Magnetic Field (EMF) direction in the

518 Northern Hemisphere. These orientations are reverse for microorganisms of the Southern 519 Hemisphere. 